EUROPEAN JOURNAL OF PURE AND APPLIED MATHEMATICS

Vol. 12, No. 1, 2019, 25-38

ISSN 1307-5543 - www.ejpam.com

Published by New York Business Global

\title{
On Determining Initial Conditions of Equations Flexural-Torsinal Vibrations of a Bar
}

Aysel T. Ramazanova

University Duisburg-Essen, Germany

\begin{abstract}
The problem of finding the initial conditions in the boundary-value problem for the system of flexural-torsional vibrations of a bar with additional conditions on the straight line is reduced to an optimal control problem and studied by the methods of optimal control theory. The gradient of the functional is calculated and using the gradient expression a necessary and sufficient optimality condition are proved.
\end{abstract}

2010 Mathematics Subject Classifications: 49j20

Key Words and Phrases: Optimal control, flexural-torsional vibrations, necessary and sufficient condition

\section{Introduction}

It is known that some problems of mathematical physics, mechanics, are described by fourth order partial equations. A tuning fork, a bar vibrations equation, a rotary shaft, oscillating motions equation and plate vibrations equation are among these equations give some references. It is imperative optimal control problems in processes described by these equations. The control connected with flexural-torsional vibrations of a bar has a great signifficance in dynamics of aircraft constructions. Therefore, the study of bar vibrations problems controls described by differential equations is necessary both from practical and theoretical point of view.

\section{Problem statement}

We consider a boundary value problem for equations of flexural-torsional vibrations of a bar, described by the system of two differential equations in the domain $Q=$ $\{0<x<l, 0<t<T\}$ with boundary and initial conditions

$$
\frac{\partial^{2}}{\partial x^{2}}\left(E(x) I(x) \frac{\partial^{2} y}{\partial x^{2}}\right)+\rho(x) A(x) \frac{\partial^{2} y}{\partial t^{2}}-\rho(x) A(x) e(x) \frac{\partial^{2} \theta}{\partial t^{2}}=f_{1}(x, t),
$$

DOI: https://doi.org/10.29020/nybg.ejpam.v12i1.3350

Email addresses: ramazanova-aysel@mail.ru 


$$
\begin{gathered}
\frac{\partial^{2}}{\partial x^{2}}\left(E(x) C_{w}(x) \frac{\partial^{2} \theta}{\partial x^{2}}\right)-G(x) C(x) \frac{\partial^{2} \theta}{\partial x^{2}}-\rho(x) A(x) e(x) \frac{\partial^{2} y}{\partial t^{2}}+ \\
+\rho(x)\left(I(x)+A(x) e^{2}(x)\right) \frac{\partial^{2} \theta}{\partial t^{2}}=f_{2}(x, t),(x, t) \in Q \\
y_{x=0}=\left.y\right|_{x=l}=0,\left.\quad \frac{\partial y}{\partial x}\right|_{x=0}=\left.\frac{\partial y}{\partial x}\right|_{x=l}=0, \quad 0 \leq \mathrm{t} \leq \mathrm{T}, \\
\left.\theta\right|_{x=0}=\left.\theta\right|_{x=l}=0,\left.\quad \frac{\partial \theta}{\partial x}\right|_{x=0}=\left.\frac{\partial \theta}{\partial x}\right|_{x=l}=0, \quad 0 \leq \mathrm{t} \leq \mathrm{T}, \\
\left.y\right|_{t=0}=0,\left.\quad \frac{\partial y}{\partial t}\right|_{t=0}=v_{1}(x), \quad 0 \leq \mathrm{x} \leq \mathrm{l}, \\
\left.\theta\right|_{t=0}=0,\left.\quad \frac{\partial \theta}{\partial t}\right|_{t=0}=w_{1}(x), \quad 0 \leq \mathrm{x} \leq 1,
\end{gathered}
$$

where $l>0, T>0$ are given numbers, $y(x, t)$ is the lateral displacement of the bar, $\theta(x, t)$ is the turning angle of the bar cross-section, $E(x)$ is the Young modulus, $I(x)$ is a polar inertia moment of the cross section with respect to its gravity center, $\rho(x)$ is a density of the bar material, $A(x)$ is the area cross section, $e(x)$ is the distance from the gravity center to the center of torsion, $C_{w}(x)$ is the sectional moment of inertia of the crosssection, $G(x)$ a shear modulus, $C(x)$ is geometrical rigidity of free torsion, $E(x) C_{w}(x)$ is the rigidity of flexural functions, $G(x) C(x)$ is the rigidity of free torsion, the functions $\left(v_{1}(x), w_{1}(x)\right) \in L_{2}(0, l) \times L_{2}(0, l)$ - to be defined.

Note that for each fixed vector function $\left(v_{1}(x), w_{1}(x)\right) \in L_{2}(0, l) \times L_{2}(0, l)$ problem (1)-(6) has a unique generalized solution from the spaces $W_{2}^{2,1}(Q)[3,5,6]$.

To determine $v(x)=\left(v_{1}(x), w_{1}(x)\right)$, we give the additional conditions

$$
\begin{aligned}
& y(x, T ; v)=\varphi_{1}(x), 0 \leq x \leq l, \\
& \theta(x, T ; v)=\varphi_{2}(x), 0 \leq x \leq l,
\end{aligned}
$$

where $\varphi_{1}(x), \varphi_{2}(x)$ - are given functions.

We reduce this problem to the following optimal control problem: it is required to find such a vector-function $\left(v_{1}(x), w_{1}(x)\right) \in L_{2}(0, l) \times L_{2}(0, l)$, that minimizes the functional

$$
J_{0}(v)=\frac{1}{2} \int_{0}^{l}\left[\left(\left(y(x, T ; v)-\varphi_{1}(x)\right)^{2}+\left(\theta(x, T ; v)-\varphi_{2}(x)\right)^{2}\right] d x\right.
$$

together with the solution of boundary value problem (1)-(6).

The function $v(x)=\left(v_{1}(x), w_{1}(x)\right)$ - is called a control. We call problem (1)-(6),(9) a reduced problem. The problem (1) - (6), (9) is regularized as follows. We introduce the functional 


$$
J_{\alpha}(v)=J_{0}(v)+\frac{\alpha}{2}\left(\left\|v_{1}\right\|_{L_{2}(0, l)}^{2}+\left\|w_{1}\right\|_{L_{2}(0, l)}^{2}\right), \alpha=\text { const }>0
$$

Now for a class of admissible controls we take a convex, closed set $U_{a d} \in L_{2}(0, l) \times$ $L_{2}(0, l)$ of vector-functions $v(x)=\left(v_{1}(x), w_{1}(x)\right)$.

Suppose that data of problem (1)-(6) satisfy the following conditions:

1) $E(x), I(x), \rho(x), A(x), e(x), C_{\omega}(x), G(x), C(x)$, are mesaurable, bounded and positive functions on the interval $[0, l]$;

2) $f_{1}, f_{2} \in L_{2}(Q), \varphi_{1}, \varphi_{2} \in L_{2}(0, l)$ - are given functions.

\section{Differentiability of functional (10)}

We show that the functional $(10)$ is differentiable in $L_{2}(0, l) \times L_{2}(0, l)$.

Introduce the following problem adjoint to the problem (1)-(6), (10):

$$
\begin{gathered}
\frac{\partial^{2}}{\partial x^{2}}\left(E(x) I(x) \frac{\partial^{2} \Psi_{1}}{\partial x^{2}}\right)+\rho(x) A(x) \frac{\partial^{2} \Psi_{1}}{\partial t^{2}}-\rho(x) A(x) e(x) \frac{\partial^{2} \Psi_{2}}{\partial t^{2}}=0, \\
(x, t) \in Q \\
\frac{\partial^{2}}{\partial x^{2}}\left(E(x) C_{w}(x) \frac{\partial^{2} \Psi_{2}}{\partial x^{2}}\right)-G(x) C(x) \frac{\partial^{2} \Psi_{2}}{\partial x^{2}}-\rho(x) A(x) e(x) \frac{\partial^{2} \Psi_{1}}{\partial t^{2}}+ \\
+\rho(x)\left(I(x)+A(x) e^{2}(x)\right) \frac{\partial^{2} \Psi_{2}}{\partial t^{2}}=0,(x, t) \in Q, \\
\left.\Psi_{1}\right|_{x=0}=\left.\Psi_{1}\right|_{x=l}=0,\left.\quad \frac{\partial \Psi_{1}}{\partial x}\right|_{x=0}=\left.\frac{\partial \Psi_{1}}{\partial x}\right|_{x=l}=0, \quad 0 \leq \mathrm{t} \leq \mathrm{T}, \\
\left.\Psi_{2}\right|_{x=0}=\left.\Psi_{2}\right|_{x=l}=0,\left.\quad \frac{\partial \Psi_{2}}{\partial x}\right|_{x=0}=\left.\frac{\partial \Psi_{2}}{\partial x}\right|_{x=l}=0,0 \leq \mathrm{t} \leq \mathrm{T}, \\
\left.\Psi_{1}\right|_{t=T}=0,\left.\right|_{t=T}=0,0 \leq \mathrm{x} \leq 1, \\
\left.\frac{\partial \Psi_{1}}{\partial t}\right|_{t=T}= \\
\left.\frac{\partial \Psi_{2}}{\partial t}\right|_{t=T}=-\frac{\left(\varphi_{1}(x)-y(x, T ; v) e(x)+\varphi_{2}(x)-\theta(x, T ; v)\right)}{\rho(x) I(x)} \\
=\frac{\left(I(x)+A(x) e^{2}(x)\right)\left(\varphi_{1}(x)-y(x, T ; v)+A(x) e(x)\left(\varphi_{2}(x)-\theta(x, T ; v)\right)\right.}{\rho(x) I(x)}
\end{gathered}
$$


We take the two admissible controls and assign them the increments $\delta v_{1} \in L_{2}(0, l)$ and $\delta w_{1} \in L_{2}(0, l)$ in such a way that, $\left(v_{1}(x)+\delta v_{1}(x), w_{1}(x)+\delta w_{1}(x) \in\right.$ $\left.U_{a d}\right)$

$$
\begin{gathered}
v(x)=\left(v_{1}(x), w_{1}(x)\right), v(x)+\delta v(x)= \\
=\left(v_{1}(x)+\delta v_{1}(x), w_{1}(x)+\delta w_{1}(x)\right) \in L_{2}(0, l) .
\end{gathered}
$$

Then the increment of the functional (10) is computed as

$$
\begin{gathered}
\Delta J_{\alpha}(v)=J_{\alpha}(v+\delta v)-J_{\alpha}(v)= \\
=\frac{1}{2} \int_{0}^{l}\left[\left(y\left(x, T ; v_{1}+\delta v_{1}, w_{1}+\delta w_{1}\right)-\varphi_{1}(x)\right)^{2}-\right. \\
-\left(y\left(x, T ; v_{1}, w_{1}\right)-\varphi_{1}(x)\right)^{2}+\left(\theta\left(x, T ; v_{1}+\delta v_{1}, w_{1}+\delta w_{1}\right)-\varphi_{2}(x)\right)^{2}- \\
\left.-\left(\theta\left(x, T ; v_{1}, w_{1}\right)-\varphi_{2}(x)\right)^{2}\right] d x+ \\
+\frac{\alpha}{2}\left(\left\|v_{1}+\delta v_{1}\right\|_{L_{2}(0, l)}^{2}-\left\|v_{1}\right\|_{L_{2}(0, l)}^{2}\right)+\frac{\alpha}{2}\left(\left\|w_{1}+\delta w_{1}\right\|_{L_{2}(0, l)}^{2}\right),
\end{gathered}
$$

where

$$
\begin{aligned}
& y(x, t ; v(x)+\delta v(x))=y(x, t ; v)+\delta y(x, t) \\
& \theta(x, t ; v(x)+\delta v(x))=\theta(x, t ; v)+\delta \theta(x, t) .
\end{aligned}
$$

Hence it follows that

$$
\begin{gathered}
\Delta J_{\alpha}(v)=\int_{0}^{l}\left[\left(y(x, T ; v)-\varphi_{1}(x)\right) \delta y(x, T)+\left(\theta(x, T ; v)-\varphi_{2}(x)\right) \delta \theta(x, T)\right] d x+ \\
+\alpha \int_{0}^{l}\left(v_{1} \delta v_{1}+w_{1} \delta w_{1}\right) d x+R
\end{gathered}
$$

where

$$
\mathrm{R}=\frac{1}{2} \int_{0}^{l}\left[(\delta y(x, T))^{2}+(\delta \theta(x, T))^{2}\right] d x+\frac{\alpha}{2}\left(\int_{0}^{l}\left(\left(\delta v_{1}\right)^{2}+\left(\delta w_{1}\right)^{2}\right) d x\right)
$$

and

$(\delta y(x, t), \delta \theta(x, t)) \in W_{2}^{2,1}(Q) \times W_{2}^{2,1}(Q)$ is the generalized solution of the

$$
\begin{gathered}
\frac{\partial^{2}}{\partial x^{2}}\left(E(x) I(x) \frac{\partial^{2} \delta y}{\partial x^{2}}\right)+\rho(x) A(x) \frac{\partial^{2} \delta y}{\partial t^{2}}- \\
-\rho(x) A(x) e(x) \frac{\partial^{2} \delta \theta}{\partial t^{2}}=0,(x, t) \in Q,
\end{gathered}
$$




$$
\begin{gathered}
\frac{\partial^{2}}{\partial x^{2}}\left(E(x) C_{w}(x) \frac{\partial^{2} \delta \theta}{\partial x^{2}}\right)-G(x) C(x) \frac{\partial^{2} \delta \theta}{\partial x^{2}}- \\
-\rho(x) A(x) e(x) \frac{\partial^{2} \delta y}{\partial t^{2}}+\rho(x)\left(I(x)+A(x) e^{2}(x)\right) \frac{\partial^{2} \delta \theta}{\partial t^{2}}=0,(x, t) \in Q, \\
\left.\delta y\right|_{x=0}=\left.\delta y\right|_{x=l}=0,\left.\frac{\partial \delta y}{\partial x}\right|_{x=0}=\left.\frac{\partial \delta y}{\partial x}\right|_{x=l}=0,0 \leq t \leq T, \\
\left.\delta \theta\right|_{x=0}=\left.\delta \theta\right|_{x=l}=0,\left.\frac{\partial \delta \theta}{\partial x}\right|_{x=0}=\left.\frac{\partial \delta \theta}{\partial x}\right|_{x=l}=0,0 \leq t \leq T, \\
\left.\delta y\right|_{t=0}=0,\left.\frac{\partial \delta y}{\partial t}\right|_{t=0}=\delta v_{1}(x), \\
\left.\delta \theta\right|_{t=0}=0,\left.\quad \frac{\partial \delta \theta}{\partial t}\right|_{t=0}=\delta w_{1}(x), \quad 0 \leq x \leq l
\end{gathered}
$$

i.e. for any function

$$
\begin{gathered}
\forall \eta_{1}=\eta_{1}(x, t), \quad \eta_{2}=\eta_{2}(x, t) \in W_{2}^{2,1}(Q), \\
\left.\eta_{1}\right|_{x=0}=\left.\eta_{1}\right|_{x=l}=0,\left.\quad \frac{\partial \eta_{1}}{\partial x}\right|_{x=0}=\left.\frac{\partial \eta_{1}}{\partial x}\right|_{x=l}=0, \quad 0 \leq \mathrm{t} \leq \mathrm{T}, \\
\left.\eta_{2}\right|_{x=0}=\left.\eta_{2}\right|_{x=l}=0,\left.\quad \frac{\partial \eta_{2}}{\partial x}\right|_{x=0}=\left.\frac{\partial \eta_{2}}{\partial x}\right|_{x=l}=0, \quad 0 \leq \mathrm{t} \leq \mathrm{T},
\end{gathered}
$$

the following integral identities are fulfilled

$$
\begin{gathered}
\iint_{Q}\left(E(x) I(x) \frac{\partial^{2} \delta y}{\partial x^{2}} \frac{\partial^{2} \eta_{1}}{\partial x^{2}}-\rho(x) A(x) \frac{\partial \delta y}{\partial t} \frac{\partial \eta_{1}}{\partial t}+\right. \\
\left.\rho(x) A(x) e(x) \frac{\partial \delta \theta}{\partial t} \frac{\partial \eta_{1}}{\partial t}\right) d x d t+ \\
+\left.\int_{0}^{l} \rho(x) A(x) \frac{\partial \delta y}{\partial t} \eta_{1}\right|_{0} ^{T} d x-\left.\int_{0}^{l} \rho(x) A(x) e(x) \frac{\partial \delta \theta}{\partial t} \eta_{1}\right|_{0} ^{T} d x=0 \\
\iint_{Q}\left(E(x) C_{w}(x) \frac{\partial^{2} \delta \theta}{\partial x^{2}} \frac{\partial^{2} \eta_{2}}{\partial x^{2}}-G(x) C(x) \frac{\partial^{2} \eta_{2}}{\partial x^{2}} \delta \theta+\right. \\
\left.+\rho(x) A(x) e(x) \frac{\partial \delta y}{\partial t} \frac{\partial \eta_{2}}{\partial t}-\rho(x)\left(I(x)+A(x) e^{2}(x)\right) \frac{\partial \delta \theta}{\partial t} \frac{\partial \eta_{2}}{\partial t}\right) d x d t- \\
\left.\int_{0}^{l} \rho(x) A(x) e(x) \frac{\partial \delta y}{\partial t} \eta_{2}\right|_{0} ^{T} d x+
\end{gathered}
$$




$$
+\left.\int_{0}^{l} \rho(x)\left(I(x)+A(x) e^{2}(x)\right) \frac{\partial \delta \theta}{\partial t} \eta_{2}\right|_{0} ^{T} d x=0 .
$$

As the functions $\left(\Psi_{1}(x, t), \Psi_{2}(x, t)\right)$ are the generalized solutions of problem (11)- (15), for any functions $g_{1}, g_{2} \in W_{2}^{2,1}(Q)$,

$$
\begin{aligned}
& \left.g_{1}\right|_{x=0}=\left.g_{1}\right|_{x=l}=0,\left.\quad \frac{\partial g_{1}}{\partial x}\right|_{x=0}=\left.\frac{\partial g_{1}}{\partial x}\right|_{x=l}=0, \quad 0 \leq \mathrm{t} \leq \mathrm{T}, \\
& \left.g_{2}\right|_{x=0}=\left.g_{2}\right|_{x=l}=0,\left.\quad \frac{\partial g_{2}}{\partial x}\right|_{x=0}=\left.\frac{\partial g_{2}}{\partial x}\right|_{x=l}=0, \quad 0 \leq \mathrm{t} \leq \mathrm{T},
\end{aligned}
$$

the following integral identities are fulfilled

$$
\begin{gathered}
\iint_{Q}\left(E(x) l(x) \frac{\partial^{2} \Psi_{1}}{\partial x^{2}} \frac{\partial^{2} g_{1}}{\partial x^{2}}-\rho(x) A(x) \frac{\partial \Psi_{1}}{\partial t} \frac{\partial g_{1}}{\partial t}+\right. \\
\left.+\rho(x) A(x) e(x) \frac{\partial \Psi_{2}}{\partial t} \frac{\partial g_{1}}{\partial t}\right) d x d t+\left.\int_{0}^{l} \rho(x) A(x) \frac{\partial \Psi_{1}}{\partial t} g_{1}\right|_{0} ^{T} d x- \\
-\left.\int_{0}^{l} \rho(x) A(x) e(x) \frac{\partial \Psi_{2}}{\partial t} g_{1}\right|_{0} ^{T} d x=0,(x, t) \in Q \\
\iint_{Q}\left(E(x) C_{w}(x) \frac{\partial^{2} \Psi_{2}}{\partial x^{2}} \frac{\partial^{2} g_{2}}{\partial x^{2}}-G(x) C(x) \frac{\partial^{2} \Psi_{2}}{\partial x^{2}} g_{2}+\right. \\
\left.+\rho(x) A(x) e(x) \frac{\partial \Psi_{1}}{\partial t} \frac{\partial g_{2}}{\partial t}-\rho(x)\left(I(x)+A(x) e^{2}(x)\right) \frac{\partial \Psi_{2}}{\partial t} \frac{\partial g_{2}}{\partial t}\right) d x d t- \\
-\left.\int_{0}^{l} \rho(x) A(x) e(x) \frac{\partial \Psi_{1}}{\partial t} g_{2}\right|_{0} ^{T} d x+ \\
+\left.\int_{0}^{l} \rho(x)\left(I(x)+A(x) e^{2}(x)\right) \frac{\partial \Psi_{2}}{\partial t} g_{2}\right|_{0} ^{T} d x=0,(x, t) \in Q .
\end{gathered}
$$

In equalities (23) and (24) instead of $\eta_{1}(x, t)$ and $\eta_{2}(x, t)$ we take $\Psi_{1}(x, t)$ and $\Psi_{2}(x, t)$, in the identities (25) and (26) instead of $g_{1}(x, t)$ and $g_{2}(x, t)$ we take $\delta y(x, t)$ and $\delta \theta(x, t)$ respectively, subtract the obtained relations and sum them. Then we have,

$$
\begin{gathered}
\int_{0}^{l}\left[\left(y(x, T ; v)-\varphi_{1}(x)\right) \delta y(x, T) d x+\left(\theta(x, T ; v)-\varphi_{2}(x)\right) \delta \theta(x, T) d x=\right. \\
=\int_{0}^{l}\left[\rho(x) A(x) \Psi_{1}(x, 0)-\rho(x) A(x) e(x) \Psi_{2}(x, 0)\right] \delta v_{1} d x+ \\
+\int_{0}^{l}\left[-\rho(x) A(x) e(x) \Psi_{1}(x, 0)+\rho(x)\left(I(x)+A(x) e^{2}(x)\right) \Psi_{2}(x, 0)\right] \delta w_{1} \mathrm{~d} \mathrm{x}
\end{gathered}
$$


Therefore from formulas (17) and (27) it follows that

$$
\begin{gathered}
\Delta J_{\alpha}(v)=\int_{0}^{l}\left[\rho(x) A(x) \Psi_{1}(x, 0)-\rho(x) A(x) e(x) \Psi_{2}(x, 0)+\alpha v_{1}\right] \delta v_{1} d x+ \\
+\int_{0}^{l}\left[-\rho(x) A(x) e(x) \Psi_{1}(x, 0)+\rho(x)\left(I(x)+A(x) e^{2}(x)\right) \cdot\right. \\
\left.\cdot \Psi_{2}(x, 0)+\alpha w_{1}\right] \delta w_{1} d x+R
\end{gathered}
$$

Next we show that

$$
\begin{gathered}
\|\delta y(x, T)\|_{L_{2(0, l)}}^{2} \leq c\left\|\delta v_{1}\right\|_{L_{2}(0, l)}^{2}, \\
\|\delta \theta(x, T)\|_{L_{2(0, l)}}^{2} \leq c\left\|\delta w_{1}\right\|_{L_{2}(0, l)}^{2} .
\end{gathered}
$$

For this purpose first we show that

$$
\begin{gathered}
\|\delta y(x, t)\|_{W_{2}^{2,1}(Q)}^{2} \leq c\left\|\delta v_{1}\right\|_{L_{2}(0, l)}^{2}, \\
\|\delta \theta(x, t)\|_{W_{2}^{2,1}(Q)}^{2} \leq c\left\|\delta w_{1}\right\|_{L_{2}(0, l)}^{2}
\end{gathered}
$$

For proving estimations (31) and (32) we apply the Faedo-Galerkin method. Let $\left\{\omega_{i}(x)\right\}_{i=1}^{\infty}$ be a fundamental system in $W_{2}^{2}(0, l)$ and

$$
\int_{0}^{l} \omega_{i}(x) \omega_{k}(x) d x= \begin{cases}1, & i=k \\ 0, & i \neq k\end{cases}
$$

We look for approximate solutions $\left(\delta y^{N}(x, t), \delta \theta^{N}(x, t)\right)$ of problem (18), (19) in the form $\delta y^{N}(x, t)=\sum_{i=1}^{N} c_{1 i}^{N}(t) \omega_{i}(x)$ and $\delta \theta^{N}(x, t)=\sum_{i=1}^{N} c_{2 i}^{N}(t) \omega_{i}(x)$ from the following relations

$$
\begin{gathered}
\int_{0}^{l}\left(E(x) I(x) \frac{\partial^{2} \delta y^{N}}{\partial x^{2}} \frac{d^{2} \omega_{p}(x)}{\partial x^{2}}+\rho(x) A(x) \frac{\partial^{2} \delta y^{N}}{\partial t^{2}} \omega_{p}(x)-\right. \\
\left.-\rho(x) A(x) e(x) \frac{\partial^{2} \delta \theta^{N}}{\partial t^{2}} \omega_{p}(x)\right) d x=0, \quad p=\overline{1, N,} \\
\int_{0}^{l}\left(E(x) C_{w}(x) \frac{\partial^{2} \delta \theta^{N}}{\partial x^{2}} \frac{d^{2} \omega_{p}(x)}{\partial x^{2}}-G(x) C(x) \delta \theta^{N} \frac{d^{2} \omega_{p}(x)}{\partial x^{2}}-\right. \\
-\rho(x) A(x) e(x) \frac{\partial^{2} \delta y^{N}}{\partial t^{2}} \omega_{p}(x)+ \\
\left.\rho(x)\left(I(x)+A(x) e^{2}(x)\right) \frac{\partial^{2} \delta \theta^{N}}{\partial t^{2}} \omega_{p}(x)\right) d x=0 p=\overline{1, N}
\end{gathered}
$$




$$
\begin{gathered}
\left.c_{1 i}^{N}\right|_{t=0}=0,\left.\frac{d c_{1 i}^{N}(t)}{d t}\right|_{t=0}=\left(v_{1}, \omega_{i}\right), \\
\left.c_{2 i}^{N}\right|_{t=0}=0,\left.\frac{d c_{2 i}^{N}(t)}{d t}\right|_{t=0}=\left(w_{1}, \omega_{i}\right), i=\overline{1, N} .
\end{gathered}
$$

Equalities (33) and (34) are the system of linear ordinary differential equations of second order with the unknowns $c_{1 i}^{N}(t)$ and $c_{2 i}^{N}(t), i=\overline{1, N}$ solved with respect to $d^{2} c_{1 i}^{N} / d t^{2}$ and $d^{2} c_{2 i}^{N} / d t^{2}$. Under the conditions on the problem data, this system is uniquely solvable under initial conditions (35) and (36), moreover $d^{2} c_{1 i}^{N} / d t^{2}, d^{2} c_{2 i}^{N} / d t^{2} \in L_{2}(0, T), i=\overline{1, N}$.

Multiplying each of the equalities (33) and (34) by its own $d c_{1 p}^{N} / d t, d c_{2 p}^{N} / d t$ and summing over $p$ from 1 to $N$ we come to the equalities

$$
\begin{gathered}
\int_{0}^{l}\left(E(x) I(x) \frac{\partial^{2} \delta y^{N}}{\partial x^{2}} \frac{\partial^{3} \delta y^{N}}{\partial x^{2} \partial t}+\rho(x) A(x) \frac{\partial^{2} \delta y^{N}}{\partial t^{2}} \frac{\partial \delta y^{N}}{\partial t}-\right. \\
\left.-\rho(x) A(x) e(x) \frac{\partial^{2} \delta \theta^{N}}{\partial t^{2}} \frac{\partial \delta y^{N}}{\partial t}\right) d x=0 \\
\int_{0}^{l}\left(E(x) C_{w}(x) \frac{\partial^{2} \delta \theta^{N}}{\partial x^{2}} \frac{\partial^{3} \delta \theta^{N}}{\partial x^{2} \partial t}-G(x) C(x) \delta \theta^{N} \frac{\partial^{3} \delta \theta^{N}}{\partial x^{2} \partial t}-\right. \\
\rho(x) A(x) e(x) \frac{\partial^{2} \delta y^{N}}{\partial t^{2}} \frac{\partial \delta \theta^{N}}{\partial t}+ \\
\left.+\rho(x)\left(I(x)+A(x) e^{2}(x)\right) \frac{\partial^{2} \delta \theta^{N}}{\partial t^{2}} \frac{\partial \delta \theta^{N}}{\partial t}\right) d x=0
\end{gathered}
$$

Suppose that $G(x) C(x)$ are independent of $x$.

Then it follows that

$$
\begin{gathered}
\frac{1}{2} \frac{d}{d t} \int_{0}^{l}\left[\left(E(x) I(x)\left(\frac{\partial^{2} \delta y^{N}}{\partial x^{2}}\right)^{2}+\rho(x) A(x)\left(\frac{\partial \delta y^{N}}{\partial t}\right)^{2}+E(x) C_{w}(x)\left(\frac{\partial^{2} \delta \theta^{N}}{\partial x^{2}}\right)^{2}+\right.\right. \\
+G C\left(\frac{\partial \delta \theta^{N}}{\partial x}\right)^{2}+\rho(x)\left(I(x)+A(x) e^{2}(x)\right)\left(\frac{\partial \delta \theta^{N}}{\partial t}\right)^{2}- \\
\left.-2 \rho(x) A(x) e(x)\left(\frac{\partial \delta y^{N}}{\partial t} \frac{\partial \delta \theta^{N}}{\partial t}\right)\right] d x=0
\end{gathered}
$$

We integrate the last equality with respect to $t$ from 0 to $t$ :

$$
\int_{0}^{l}\left[\left(E(x) I(x)\left(\frac{\partial^{2} \delta y^{N}}{\partial x^{2}}\right)^{2}+\rho(x) A(x)\left(\frac{\partial \delta y^{N}}{\partial t}\right)^{2}+E(x) C_{w}(x)\left(\frac{\partial^{2} \delta \theta^{N}}{\partial x^{2}}\right)^{2}+\right.\right.
$$




$$
\begin{gathered}
+G C\left(\frac{\partial \delta \theta^{N}}{\partial x}\right)^{2}+\rho(x)\left(I(x)+A(x) e^{2}(x)\right)\left(\frac{\partial \delta \theta^{N}}{\partial t}\right)^{2}- \\
\left.-2 \rho(x) A(x) e(x)\left(\frac{\partial \delta y^{N}}{\partial t} \frac{\partial \delta \theta^{N}}{\partial t}\right)\right] d x= \\
=\int_{0}^{l}\left[\rho(x) A(x)\left(\frac{\partial \delta y^{N}(x, 0)}{\partial t}\right)^{2}+\rho(x)\left(I(x)+A(x) e^{2}(x)\right)\left(\frac{\partial \delta \theta^{N}(x, 0)}{\partial t}\right)^{2}-\right. \\
\left.-2 \rho(x) A(x) e(x)\left(\frac{\partial \delta y^{N}(x, 0)}{\partial t} \frac{\partial \delta \theta^{N}(x, 0)}{\partial t}\right)\right] d x
\end{gathered}
$$

In equality (40) we make some transformations:

$$
\begin{gathered}
\int_{0}^{l}\left[\left(E(x) I(x)\left(\frac{\partial^{2} \delta y^{N}}{\partial x^{2}}\right)^{2}+\rho(x) A(x)\left(\frac{\partial \delta y^{N}}{\partial t}\right)^{2}+E(x) C_{w}(x)\left(\frac{\partial^{2} \delta \theta^{N}}{\partial x^{2}}\right)^{2}+\right.\right. \\
+G C\left(\frac{\partial \delta \theta^{N}}{\partial x}\right)^{2}+\rho(x)\left(I(x)+A(x) e^{2}(x)\right)\left(\frac{\partial \delta \theta^{N}}{\partial t}\right)^{2}- \\
\left.-\rho(x) A(x) e(x)\left(\left(\frac{\partial \delta y^{N}}{\partial t}\right)^{2}+\left(\frac{\partial \delta \theta^{N}}{\partial t}\right)^{2}\right)\right] d x \leq \\
\leq \int_{0}^{l}\left[\rho(x) A(x)\left(\frac{\partial \delta y^{N}(x, 0)}{\partial t}\right)^{2}+\rho(x)\left(I(x)+A(x) e^{2}(x)\right)\left(\frac{\partial \delta \theta^{N}(x, 0)}{\partial t}\right)^{2}+\right. \\
\left.+\rho(x) A(x) e(x)\left(\left(\frac{\partial \delta y^{N}(x, 0)}{\partial t}\right)^{2}+\left(\frac{\partial \delta \theta^{N}(x, 0)}{\partial t}\right)^{2}\right)\right] d x
\end{gathered}
$$

It's clear that,

$$
\begin{gathered}
\int_{0}^{l}\left|\frac{\partial \delta y^{N}(x, 0)}{\partial t}\right|^{2} d x \leq \int_{0}^{l}\left|\sum_{i=1}^{N}\left(\delta v_{1}, \omega_{i}\right) \omega_{i}(x)\right|^{2} d x \leq \\
\leq c \sum_{i=1}^{N}\left|\delta v_{1 i}\right|^{2} \leq c \sum_{i=1}^{\infty}\left|\delta v_{1 i}\right|^{2} \leq c\left\|\delta v_{1}\right\|_{L_{2}(0, l)}^{2}, \\
\int_{0}^{l}\left|\frac{\partial \delta \theta^{N}(x, 0)}{\partial t}\right|^{2} d x \leq \int_{0}^{l}\left|\sum_{i=1}^{N}\left(\delta w_{1}, \omega_{i}\right) \omega_{i}(x)\right|^{2} d x \leq
\end{gathered}
$$




$$
\leq c \sum_{i=1}^{N}\left|\delta w_{1 i}\right|^{2} \leq c \sum_{i=1}^{\infty}\left|\delta w_{1 i}\right|^{2} \leq c\left\|\delta w_{1}\right\|_{L_{2}(0, l)}^{2},
$$

where $\delta v_{1 i}=\int_{0}^{l} \delta v_{1}(x) \omega_{i}(x) d x, \delta w_{1 i}=\int_{0}^{l} \delta w_{1}(x) \omega_{i}(x) d x$ are Fourier coefficients of the functions $\delta v_{1}(x), \delta w_{1}(x)$. From (41), (53), (43) we get:

$$
\begin{gathered}
\int_{0}^{l}\left[\left(E(x) I(x)\left(\frac{\partial^{2} \delta y^{N}}{\partial x^{2}}\right)^{2}+\rho(x) A(x)\left(\frac{\partial \delta y^{N}}{\partial t}\right)^{2}+E(x) C_{w}(x)\left(\frac{\partial^{2} \delta \theta^{N}}{\partial x^{2}}\right)^{2}+\right.\right. \\
+G C\left(\frac{\partial \delta \theta^{N}}{\partial x}\right)^{2}+\rho(x)\left(I(x)+A(x) e^{2}(x)\right)\left(\frac{\partial \delta \theta^{N}}{\partial t}\right)^{2}- \\
\left.-\rho(x) A(x) e(x)\left(\left(\frac{\partial \delta y^{N}}{\partial t}\right)^{2}+\left(\frac{\partial \delta \theta^{N}}{\partial t}\right)^{2}\right)\right] d x \leq \\
\leq \int_{0}^{l}\left[\rho(x) A(x)\left(\frac{\partial \delta y^{N}(x, 0)}{\partial t}\right)^{2}+\rho(x)(I(x)+\right. \\
+A(x) e^{2}(x)\left(\frac{\partial \delta \theta^{N}(x, 0)}{\partial t}\right)^{2}+ \\
\left.+\rho(x) A(x) e(x)\left(\left(\frac{\partial \delta y^{N}(x, 0)}{\partial t}\right)^{2}+\left(\frac{\partial \delta \theta^{N}(x, 0)}{\partial t}\right)^{2}\right)\right] d x \leq \\
\leq c\left(\left\|\delta v_{1}\right\|_{L_{2}(0, l)}^{2}+\left\|\delta w_{1}\right\|_{L_{2}(0, l)}^{2}\right)
\end{gathered}
$$

Assume that $1-e(x) \geq \alpha_{0}>0, I(x)+A(x) e(x)(e(x)-1) \geq \alpha_{1}>0$, $\forall x \in[0, l]$, where $\alpha_{0}, \alpha_{1}>0$ - are the given numbers.

Since $E(x), I(x), A(x), C_{\omega}(x), \rho(x)$ are positive functions on the segment $[0, l]$, by equivalence of the norms in the space $W_{2}^{2}(0, l)$, from the last inequality by means of elementary transformations we get:

$$
\begin{aligned}
& \int_{0}^{l}\left[\left(\delta y^{N}(x, t)\right)^{2}+\left(\frac{\partial \delta y^{N}(x, t)}{\partial t}\right)^{2}+\left(\frac{\partial \delta y^{N}(x, t)}{\partial x}\right)^{2}+\left(\frac{\partial^{2} \delta y^{N}(x, t)}{\partial x^{2}}\right)^{2}+\right. \\
& \left.+\left(\delta \theta^{N}(x, t)\right)^{2}+\left(\frac{\partial \delta \theta^{N}(x, t)}{\partial t}\right)^{2}+\left(\frac{\partial \delta \theta^{N}(x, t)}{\partial x}\right)^{2}+\left(\frac{\partial^{2} \delta \theta^{N}(x, t)}{\partial x^{2}}\right)^{2}\right] d x \leq
\end{aligned}
$$




$$
\begin{gathered}
\leq c \int_{0}^{t} \int_{0}^{l}\left[\left(\delta y^{N}(x, s)\right)^{2}+\left(\frac{\partial \delta y^{N}(x, s)}{\partial t}\right)^{2}+\left(\frac{\partial \delta y^{N}(x, s)}{\partial x}\right)^{2}+\left(\frac{\partial^{2} \delta y^{N}(x, s)}{\partial x^{2}}\right)^{2}+\right. \\
\left.+\left(\delta \theta^{N}(x, s)\right)^{2}+\left(\frac{\partial \delta \theta^{N}(x, s)}{\partial t}\right)^{2}+\left(\frac{\partial \delta \theta^{N}(x, s)}{\partial x}\right)^{2}+\left(\frac{\partial^{2} \delta \theta^{N}(x, s)}{\partial x^{2}}\right)^{2}\right] d x d s+ \\
+c\left(\left\|\delta v_{1}\right\|_{L_{2}(0, l)}^{2}+\left\|\delta w_{1}\right\|_{L_{2}(0, l)}^{2}\right)
\end{gathered}
$$

Applying the Gronuoll lemma, we have:

$$
\begin{gathered}
\int_{0}^{l}\left[\left(\delta y^{N}(x, t)\right)^{2}+\left(\frac{\partial \delta y^{N}(x, t)}{\partial t}\right)^{2}+\left(\frac{\partial \delta y^{N}(x, t)}{\partial x}\right)^{2}+\left(\frac{\partial^{2} \delta y^{N}(x, t)}{\partial x^{2}}\right)^{2}+\right. \\
\left.+\left(\delta \theta^{N}(x, t)\right)^{2}+\left(\frac{\partial \delta \theta^{N}(x, t)}{\partial t}\right)^{2}+\left(\frac{\partial \delta \theta^{N}(x, t)}{\partial x}\right)^{2}+\left(\frac{\partial^{2} \delta \theta^{N}(x, t)}{\partial x^{2}}\right)^{2}\right] d x \leq \\
\leq c\left(\left\|\delta v_{1}\right\|_{L_{2}(0, l)}^{2}+\left\|\delta w_{1}\right\|_{L_{2}(0, l)}^{2}\right) \forall t \in[0, T] .
\end{gathered}
$$

From the last inequality it follows that

$$
\begin{gathered}
\int_{0}^{T} \int_{0}^{l}\left[\left(\delta y^{N}(x, t)\right)^{2}+\left(\frac{\partial \delta y^{N}(x, t)}{\partial t}\right)^{2}+\left(\frac{\partial \delta y^{N}(x, t)}{\partial x}\right)^{2}+\left(\frac{\partial^{2} \delta y^{N}(x, t)}{\partial x^{2}}\right)^{2}+\right. \\
\left.+\left(\delta \theta^{N}(x, t)\right)^{2}+\left(\frac{\partial \delta \theta^{N}(x, t)}{\partial t}\right)^{2}+\left(\frac{\partial \delta \theta^{N}(x, t)}{\partial x}\right)^{2}+\left(\frac{\partial^{2} \delta \theta(x, t)}{\partial x^{2}}\right)^{2}\right] d x \leq \\
\leq c\left(\left\|\delta v_{1}\right\|_{L_{2}(0, l)}^{2}+\left\|\delta w_{1}\right\|_{L_{2}(0, l)}^{2}\right) .
\end{gathered}
$$

From the sequence $\left(\delta y^{N}, \delta \theta^{N}\right)$ we can choose a subsequence weakly convergent in $W_{2}^{2,1}(Q) \times$ $W_{2}^{2,1}(Q)$ to some element $(\delta y, \delta \theta) \in W_{2}^{2,1}(Q) \times W_{2}^{2,1}(Q)$. By virtue of weak lower semicontinuity of the norm in the Hilbert space, we get from (47) that for $\delta y(x, t)$ and $\delta \theta(x, t)$ the following estimation is valid

$$
\|\delta y\|_{W_{2}^{2,1}(Q)}^{2}+\|\delta \theta\|_{W_{2}^{2,1}(Q)}^{2} \leq c\left(\left\|\delta v_{1}\right\|_{L_{2}(0, l)}^{2}+\left\|\delta w_{1}\right\|_{L_{2}(0, l)}^{2}\right) .
$$

Hence the estimate (31) and (32).

Since $W_{2}^{2,1}(Q)$ is boundedly imbedded in $L_{2}(0, T)[6$, pp. 73-74], hence it follows that 


$$
\begin{gathered}
\|\delta y(x, T)\|_{L_{2}(0, l)}^{2} \leq c_{1}\|\delta y\|_{W_{2}^{2,1}(Q)}^{2} \leq c\left(\left\|\delta v_{1}\right\|_{L_{2}(0, l)}^{2}+\left\|\delta w_{1}\right\|_{L_{2}(0, l)}^{2}\right), \\
\|\delta \theta(x, T)\|_{L_{2}(0, l)}^{2} \leq c_{2}\|\delta \theta\|_{W_{2}^{2,1}(Q)}^{2} \leq c\left(\left\|\delta v_{1}\right\|_{L_{2}(0, l)}^{2}+\left\|\delta w_{1}\right\|_{L_{2}(0, l)}^{2}\right) .
\end{gathered}
$$

It is easy to show that $(\delta y, \delta \theta)$ is the generalized solution of problem (18)-(19) [6, pp. 210-215].

Thus, from (48) we find that

$$
\begin{gathered}
\mathrm{R}=\frac{1}{2} \int_{0}^{l}\left[(\delta y(x, T))^{2}+(\delta \theta(x, T))^{2}\right] d x+\frac{\alpha}{2}\left(\int_{0}^{l}\left(\left(\delta v_{1}\right)^{2}+\left(\delta w_{1}\right)^{2}\right) d x\right) \leq \\
\leq c\left(\left\|\delta v_{1}\right\|_{L_{2}(0, l)}^{2}+\left\|\delta w_{1}\right\|_{L_{2}(0, l)}^{2}\right) .
\end{gathered}
$$
to

Thus, from (28) and (49) it follows that the differential of the functional $J(v)$ is equal

$$
\begin{gathered}
\quad\left\langle J^{\prime}(v), \delta v\right\rangle=\int_{0}^{l}\left[\rho(x) A(x) \Psi_{1}(x, 0)-\rho(x) A(x) e(x) \Psi_{2}(x, 0)+\alpha v_{1}\right] \delta v_{1} \mathrm{dx}+ \\
+\left[-\rho(x) A(x) e(x) \Psi_{1}(x, 0)+\rho(x)\left(I(x)+A(x) e^{2}(x)\right) \Psi_{2}(x, 0)+\alpha w_{1}\right] \delta w_{1} \mathrm{dx} .
\end{gathered}
$$

\section{Necessary and sufficient condition of optimality}

Theorem 1. For the control $v(x)=\left(v_{1}^{0}(x), w_{1}^{0}(x)\right)$ to be an optimal control in problem (1)-(6), (10) it is necessary and sufficient that

$$
\begin{gathered}
\int_{0}^{l}\left[\rho(x) A(x) \quad \Psi_{1}(x, 0)-\rho(x) A(x) e(x) \Psi_{2}(x, 0)+\alpha v_{1}\right]\left(v_{1}(x)-v_{1}^{0}(x)\right) \mathrm{d} \mathrm{x}+ \\
+\left[\int_{0}^{l}-\rho(x) A(x) e(x) \Psi_{1}(x, 0)+\rho(x)\left(I(x)+A(x) e^{2}(x)\right) \Psi_{2}(x, 0)+\alpha w_{1}\right] \times \\
\times\left(w_{1}(x)-w_{1}^{0}(x)\right) \mathrm{dx} \geq 0, \forall v=\left(v_{1}, w_{1}\right) \in U_{a d} .
\end{gathered}
$$

Proof. Let $v_{0}(x)=\left(v_{1}^{0}(x), w_{1}^{0}(x)\right)$ - to be an optimal control in problem (1)-(6), (10). As $U_{a d^{-}}$is a convex set in $L_{2}(0, l) \times L_{2}(0, l)$, by virtue of the known theorem from [7, pp. 28],

$$
\left\langle J^{\prime}(v), v-v_{0}\right\rangle \geq 0, \forall v \in U_{a d} .
$$


From the last inequality we get necessity. As problem (1)-(6), (10) is a linear-quadratic, the obtained condition is a sufficient condition as well for the optimality of the control $v_{0}(x)$.

Conclusion: In this paper, the inverse problem of determining the right-hand sides of the flexural-torsional vibrations of a rod is considered. This problem is reduced to the problem of optimal control. The gradient of the functional is calculated and, using the gradient expression, a necessary and sufficient optimality condition is proved.

Example 1. We consider a boundary value problem for equations of flexural-torsional vibrations of a bar, described by the system of two differential equations in the domain $Q=\{0<x<1,0<t<1\}$

$$
\begin{gathered}
\frac{\partial^{4} y}{\partial x^{4}}+4 \frac{\partial^{2} y}{\partial t^{2}}-2 \frac{\partial^{2} \theta}{\partial t^{2}}=f_{1}(x, t), \\
\frac{\partial^{4} \theta}{\partial x^{4}}-\frac{\partial^{2} \theta}{\partial x^{2}}-2 \frac{\partial^{2} y}{\partial t^{2}}+3 \frac{\partial^{2} \theta}{\partial t^{2}}=f_{2}(x, t), \quad(x, t) \in \mathrm{Q} \\
y_{x=0}=\left.y\right|_{x=1}=0,\left.\quad \frac{\partial y}{\partial x}\right|_{x=0}=\left.\frac{\partial y}{\partial x}\right|_{x=1}=0, \quad 0 \leq \mathrm{t} \leq \mathrm{T}, \\
\left.\theta\right|_{x=0}=\left.\theta\right|_{x=1}=0,\left.\quad \frac{\partial \theta}{\partial x}\right|_{x=0}=\left.\frac{\partial \theta}{\partial x}\right|_{x=1}=0, \quad 0 \leq \mathrm{t} \leq \mathrm{T}, \\
\left.y\right|_{t=0}=0,\left.\quad \frac{\partial y}{\partial t}\right|_{t=0}=v_{1}(x), \\
\left.\theta\right|_{t=0}=0,\left.\quad \frac{\partial \theta}{\partial t}\right|_{t=0}=v_{2}(x), \\
f_{1}(x, t)=24 t, \quad f_{2}(x, t)=44 t+24 t x-24 t x^{2} .
\end{gathered}
$$

In the special case, the coefficients of equations (51)-(52) were taken in the from: $E=$ $\frac{1}{2}, \quad I=2, \quad \rho=1, \quad e=\frac{1}{2}, \quad A=4, \quad C_{w}=2, \quad G=1, \quad C=1$.

In order to determine $v(x)=\left(v_{1}(x), v_{2}(x)\right)$, we give the additional conditions:

$$
\begin{aligned}
& y\left(x, \frac{1}{2}, v\right)=\frac{x^{2}(1-x)^{2}}{2} \\
& \theta\left(x, \frac{1}{2}, v\right)=x^{2}(1-x)^{2} .
\end{aligned}
$$

In this special case the functional (9) has the form

$$
J_{0}(v)=\frac{1}{2} \int_{0}^{1}\left[\left(y\left(x, \frac{1}{2}, v\right)-\frac{x^{2}(1-x)^{2}}{2}\right)^{2}+\left(\theta\left(x, \frac{1}{2}, v\right)-x^{2}(1-x)^{2}\right)^{2}\right] d x .
$$


It is easy to verify that

$$
y(x, t)=t x^{2}(1-x)^{2}, \theta(x, t)=2 t x^{2}(1-x)^{2}
$$

and

$$
\underset{v \in L_{2}(0,1) \times L_{2}(0,1)}{\inf J(v)}=\min _{v \in L_{2}(0,1) \times L_{2}(0,1)}=0,
$$

and the minimum of the functional $J(v)$ is attained for $v=v_{0}(x)=\left(v_{1}^{0}(x), v_{2}^{0}(x)\right)$ $=\left(x^{2}(1-x)^{2}, 2 x^{2}(1-x)^{2}\right)$.

In this case necessary and sufficient condition (28) is fulfilled by itself, when $\alpha=0$.

\section{References}

[1] J.L. Arman, Application of theory of optimal control of distributed parameters systems to construction optimization problems. Moscow, Mir, 1977.

[2] A.G. Butkovsky, A.I. Egorov, K.A. Lurie, Optimal Control of Distributed Systems.SIAM J. Control 6 (1968), no. 3, 437-476.

[3] A.Z. Ishmukhametov, Stability and approximation of optimal control of distributed parameters systems. Computing center of RAS, 2001.

[4] V. Komkov, The Optimal control theory of a transverse vibrations of a beam. SIAMJ. Control 6 (1968), no. 3, 401-421.

[5] F.E. Lomovtsev, N.A. Yurchuk, Cauchy problem for second order hyperbolic differential-operator equations, Diff. uravn. 12 (1976), no.12, 2242-2250.

[6] O.A. Ladyzhenskaya, Boundary value problems of mathematical physics. Moscow:Nauka, 1973.

[7] F.P. Vasil'ev, Methods for solving extrum problems. Moscow: Nauka 42, (2016), No 2, 174-187

[8] A.T.Ramazanova, G.F.Kuliev, On Finding the Right-Hand Sides of Equations of Flexural-Torsional Vibrations of a Bar, Journal of Automation and Information Sciences 48 (8), (2016). 